\title{
The Role of Surgery for Asymptomatic Primary Tumors in Unresectable Stage IV Colorectal Cancer
}

\author{
Young Wan Kim, Ik Yong Kim \\ Department of Surgery, Yonsei University Wonju College of Medicine, Wonju, Korea
}

There are still debates regarding the appropriate primary treatment policy for asymptomatic primary colorectal lesions in cases of unresectable metastatic colorectal cancer. Even though there are patients with asymptomatic primary tumors when starting chemotherapy, those patients may still undergo surgery due to complications related to primary tumors in the middle of chemotherapy; therefore, controversy exists regarding surgical resection of primary colorectal lesions in cases where symptoms are absent when making a diagnosis. Thus, based on the published literature, we discuss opinions that prefer first-line surgery for primary tumors as well as opinions favoring first-line chemotherapy for treating unresectable synchronous metastatic colorectal cancer. Although the upfront chemotherapy including targeted agents is suggested as an effective treatment in recent years, the first line surgery has been a preferred treatment for decades. The first line surgery is beneficial to prolong the survival duration given the retrospective analysis of randomized trial data. So far, no prospective comparison study has only focused on the first-line treatment modality; thus, future clinical studies focusing on the survival duration and the quality of life should be performed as soon as possible. Furthermore, at this point, multidisciplinary team approaches would be helpful in finding the appropriate therapy. Regardless of symptoms, the performance status and the tumor burden should be taken into consideration as well. In case of surgical resection, minimally invasive surgery, such as laparoscopic surgery, is recommended.

\section{Keywords: Colorectal cancer; Unresectable metastases; Resection; Primary tumour; Chemotherapy}

\section{INTRODUCTION}

Colorectal cancer is the third most common cancer after lung cancer and breast cancer worldwide; according to the report by World Health Organization (WHO), there were 1,160,000 newlydiagnosed patients in 2007, which was $9.4 \%$ of total cancer occurrence [1]. In Korea, colorectal cancer was found to be the third most common cancer after thyroid cancer and gastric cancer, and in 2009 , there were 24,986 new patients and 7,051 patients who died due to colorectal cancer [2].

Received: March 5, 2013 - Accepted: April 1, 2013

Correspondence to: Ik Yong Kim, M.D.

Department of Surgery, Yonsei University Wonju College of Medicine,

20 Ilsan-ro, Wonju 220-701, Korea

Tel: +82-33-741-0573, Fax: +82-33-744-6604

E-mail: iykim@yonsei.ac.kr

(c) 2013 The Korean Society of Coloproctology

This is an open-access article distributed under the terms of the Creative Commons Attribution NonCommercial License (http://creativecommons.org/licenses/by-nc/3.0) which permits unrestricted noncommercial use, distribution, and reproduction in any medium, provided the original work is properly cited.
Metastatic colorectal cancer (mCRC) is TNM stage IV cancer, and approximately $20 \%$ to $25 \%$ of colorectal cancer patients have distant metastasis at their initial diagnosis; also, metastasis is known to occur in about $50 \%$ of patients eventually. So far, colorectal cancer treatment has been primarily based on surgery. Radiation and chemotherapy have been used as adjuvant treatment to reduce the risk of metastasis and recurrence [3]. Therefore, the first step is to decide on resections of the primary tumor, as well as distant metastases, in patients with synchronous mCRC. In cases where the resection of a distant metastasis is not possible, either the primary tumor resection will be performed first, followed by chemotherapy or chemotherapy will be performed first. If patients present with clinical symptoms involving in primary tumors, such as severe bleeding, obstruction, perforation, and pain, the primary tumors should be removed.

Debates are still ongoing regarding the appropriate primary treatment policy for asymptomatic primary colorectal lesions in unresectable mCRC. Even though there are patients with asymptomatic primary tumors when starting chemotherapy, surgery may still be performed due to primary-tumor-related complica- 
tions in the midst of chemotherapy; therefore, controversy exists regarding surgical removal of primary colorectal lesions in the absence of symptoms when making a diagnosis.

According to the guidelines provided by the National Comprehensive Cancer Network, resection of a primary tumor is recommended only if patients present with symptoms such as obstruction, bleeding, and perforation while combination chemotherapy is suggested if there are no symptoms [4]. In spite of this, traditionally, the removal of the primary tumor has been favored, and in United States (US), 17,658 out of 26,754 patients (66\%) with mCRC between 1998 and 2000 underwent resections for primary tumors based on the data from Surveillance, Epidemiology, and End Results (SEER) [5].

Justifications for a preference to resect asymptomatic primary tumors are a potentially better survival rate expected and fewer complications and mortalities after surgery as emergent surgery can be avoided [6-10]. Furthermore, potential side effects, such as weight loss, pain, malnutrition due to obstruction, an anemia caused by bleeding, can be prevented [11], and tumor staging is more accurate as metastatic lesions are confirmed in the abdominal cavity when performing a palliative resection, which is an additional benefit of resecting of primary tumors [12]. Lowering the tumor burden maximizes the benefit of chemotherapy and increases the potential for surgery with a curative intent [7].

On the other hand, some may not prefer the resection of the primary tumor because it is not clear that a resection of the primary tumor will improve the survival rate and the quality of life [13-15] and because there have been no appropriate comparison studies through randomized studies $[16,17]$. In addition, the association between the resection of the primary tumors and an improved survival rate should be further investigated because of the advances in chemotherapies for colorectal cancer via the introduction of targeted agents (e.g., bevacizumab and cetuximab) in the mid-2000s and of a new generation of cytotoxic agents (e.g., irinotecan and oxaliplatin), in addition to 5-fluorouracil (5-FU). Thus, the authors discussed in regard to opinions that preferred first line surgery for primary tumor as well as opinions favored the first line chemotherapy in unresectable synchronous $\mathrm{mCRC}$ based on the published literature and introduced clinical studies currently ongoing (Table 1).

\section{TREATMENT STRATEGIES FOR SYNCHRONOUS METASTATIC COLORECTAL CANCER}

Multidisciplinary team discussion is critical to determine the appropriate treatments for mCRC. In stage IV colorectal cancer, there are not many cases in which a curative resection of the primary tumor and metastatic lesions (e.g., liver, lung, and distant lymph-node metastases or peritoneal metastases) is selected at the first diagnosis. However, complete recovery is possible if resectable metastatic lesions and the primary colorectal cancer are entirely removed from the patients; thus, determining appropriate patients for either surgery or chemotherapy is very important.

Resectability of mCRC can be classified into resectable metastatic group, potentially resectable, and unresectable metastatic group. In patients with synchronous MCRC, there are a number of options for surgery and chemotherapies (radiation therapy), depending upon the location of the primary tumors (either colon or rectum) and the progress of tumor metastasis. Resectability should be determined by multidisciplinary team approaches via comprehensive discussion between colorectal surgeons, hepato-

Table 1. Summary of study characteristics

\begin{tabular}{|c|c|c|c|c|c|}
\hline Characteristic & Investigator & Year of publication & Study period & Study design & Primary tumor \\
\hline \multirow[t]{8}{*}{ Favoring resection } & Ruo et al. [10] & 2003 & $1996-1999$ & Retrospective single center study & Colon \& rectum \\
\hline & Galizia et al. [7] & 2008 & 1995-2005 & Retrospective single center study (case-matched) & Colon \& rectum \\
\hline & Kaufman et al. [18] & 2008 & 1998-2003 & Retrospective single center study & Colon \& rectum \\
\hline & Bajwa et al. [19] & 2009 & 1999-2005 & Retrospective single center study & Colon \& rectum \\
\hline & Venderbosch et al. [22] & 2011 & 2003-2004 & Retrospective analysis of randomized trials (CAIR0) & Colon \& rectum \\
\hline & & & 2005-2006 & Retrospective analysis of randomized trials (CAIR02) & Colon \& rectum \\
\hline & Karoui et al. [20] & 2011 & 1998-2007 & Retrospective multicenter study & Colon \\
\hline & Ferrand et al. [23] & 2013 & 1997-2001 & Retrospective analysis of randomized trials (FFCD 9601) & Colon \& rectum \\
\hline \multirow[t]{6}{*}{ Favoring nonresection } & Scoggins et al. [44] & 1999 & 1985-1997 & Retrospective single center study & Colon \& rectum \\
\hline & Tebbutt et al. [43] & 2003 & 1990-2000 & Retrospective single center study & Colon \& rectum \\
\hline & Michel et al. [9] & 2004 & 1996-1999 & Retrospective single center study & Colon \& rectum \\
\hline & Benoist et al. [12] & 2005 & 1997-2002 & Retrospective single center study (case-matched) & Colon \& rectum \\
\hline & Seo et al. [52] & 2010 & $2001-2008$ & Retrospective single center study & Colon \& rectum \\
\hline & McCahill et al. [53] & 2012 & 2006-2009 & Prospective, multicenter phase II trial & Colon \\
\hline
\end{tabular}

CAIRO, CApecitabine, IRinotecan, and Oxaliplatin; FFCD, Fédération Francophone de Cancérologie Digestive. 
biliary surgeons, urologists, gynecologists, and thoracic surgeons. Although patients may not be candidates for a resection on the first diagnosis, later they may be reclassified into the potentially resectable group or the resectable metastatic group after combination chemotherapy. Such cases would be identified as the potentially resectable metastatic group, and chemotherapy including targeted agents would be recommended from the first diagnosis. If removing the primary and metastatic lesions is considered impossible owing to multiple metastases, treatment strategies would be established for palliative chemotherapy to extend survival duration, improve symptoms, and maintain the quality of life.

Traditionally, the objective for unresectable colorectal cancer treatment has been to prolong the survival duration and improve the quality of life, but since the 2000s, the outcome of treatment has improved, new anticancer drugs have been developed, advances in combination chemotherapy and operation techniques have been made, and multidisciplinary team discussions have been emphasized. Thus, selection of an appropriate treatment approach and the establishment of proper objectives for the therapy (e.g., survival duration extension, complete cancer cure, improvement of cancer-related symptoms, or maintenance of the quality of life) have become more significant.

\section{THE OPINION IN FAVOR OF FIRST-LINE SURGERY IN UNRESECTABLE SYNCHRONOUS METASTATIC COLORECTAL CANCER}

The resection of the primary tumor in $\mathrm{mCRC}$ has been favored as it prevents complications (e.g., obstruction, perforation, and bleeding) occurring with primary tumor growth and improves the quality of life of patients. In many cases, studies were not randomized clinical studies, but were mostly retrospective analyses conducted at single institutes. Although many studies showed that actual resection of the primary tumor prolonged the median survival duration, making an objective comparison was difficult and a high likelihood of selection bias existed as the types of chemotherapy regimen and introduction of chemotherapy after the resection were not consistent.

Ruo et al. [10] analyzed the survival rate for patients with unresectable asymptomatic stage IV colorectal cancer. Of the total 209 patients, the first-line surgery group and the first-line chemotherapy group included 82 and 127 patients, respectively. In the comparison of patients' characteristics, right colon cancer was predominant in the first-line surgery group and a single metastatic lesion was predominant in the patients with liver metastasis only. When it comes to the survival duration, there was a statistical difference between the groups; the survival durations of the first-line surgery group and the first-line chemotherapy group were 16 months and 9 months, respectively $(\mathrm{P}<0.001)$. The extent of hepatic metastasis was also an important prognosis factor in the multivariate analysis.

Galizia et al. [7] analyzed 65 colorectal cancer patients with un- resectable hepatic metastasis. The numbers of patients in the firstline surgery group and the first-line chemotherapy group were 42 and 23, respectively. Although there was no specific difference in patients' characteristics, the initiation of chemotherapy in the first-line surgery group was 35 days, which was 8 days later compared to that of the first-line chemotherapy group. However, there was a significant difference in survival duration between those groups; the survival durations of the first-line surgery group and the first-line chemotherapy group were 15.2 months and 12.3 months, respectively $(P=0.003)$. In the multivariate analysis regarding the survival rate, resection of primary tumors and less than $50 \%$ hepatic metastasis were significant prognosis factors if the performance status was satisfactory.

Kaufman et al. [18] investigated 185 stage IV colorectal cancer patients and found the survival duration in the first-line surgery group was 22 months, which was 3 months longer than that of the first-line chemotherapy group ( $\mathrm{P}<0.0001)$; further, the survival duration of the surgery plus chemotherapy group was 30 months, which was significantly longer than that of the group with chemotherapy only (15 months, $\mathrm{P}<0.0004)$. Therefore, it was concluded that primary tumor resection was beneficial to improve the survival rate in the investigated cases of colorectal cancer.

In another study, Bajwa et al. [19] analyzed 67 colorectal cancer patients with unresectable hepatic metastasis. There were 32 and 35 patients in the first-line surgery group and the first-line chemotherapy group, respectively. The survival durations of the firstline surgery group and the first-line chemotherapy group were 14 months and 6 months, respectively, which were significantly different $(\mathrm{P}=0.005)$. The occurrence of complications after the surgery and the mortality rate were $25 \%$ and $3 \%$, respectively. In the results of the prognosis factor analysis, surgical resection and positive responses to chemotherapy were favorable prognosis factors while proximal colon cancer and multiple primary cancers were unfavorable prognosis factors.

Karoui et al. [20] performed a retrospective multi-institutional analysis of patients with unresectable stage IV colon cancer utilizing the data from 6 hospitals. The authors investigated the association between the resection of the primary tumor and overall survival and only analyzed patients with favorable (Eastern Cooperative Oncology Group [ECOG] performance status 0-1); further, recent targeted therapy drugs were included for the chemotherapy excluding rectal cancer. Of the total 208 patients, the first-line surgery group included 85 patients while the first-line chemotherapy group included 123 patients. No difference in patients' characteristics was observed between the two groups whereas overall survival was significantly different; the overall survivals of the firstline surgery group and the first-line chemotherapy were 30.7 and 21.9 months, respectively, indicating a significant difference $(\mathrm{P}=$ 0.031). According to the multivariate analysis, resection of primary tumors, cases where primary resection was available after first-line chemotherapy, well-differentiated tumors, cases with hepatic metastasis only, and cases in which patients were introduced 
to an anti-vascular endothelial growth factor (VEGF) agent were found to be prognosis factors and had a positive effect on the survival rate. The occurrence rate of complications related with primary tumors was 19\% $(n=23)$ : 21 patients with obstruction and 2 patients with perforation. Of the 21 patients with obstruction, 8 patients were treated with stent insertion while 13 patients were treated with a colectomy without stoma formation. Because antiVEGF agent has been reported to increase the risk of perforation [21], the two patients with perforation were not treated with antiVEGF agent, but were treated with resection of the primary tumor along with stoma formation. Thus, the authors suggested that application of anti-VEGF agent followed by resection of the primary tumor would be beneficial if patients present with favorable physical conditions, well-differentiated tumors, and hepatic metastases only even though there is a lack of evidence regarding the improvement of the survival rate in the first-line surgery group.

Recently, Venderbosch et al. [22] and Ferrand et al. [23] reported the results from a retrospective analysis of prospective, randomized clinical studies. Venderbosch et al. [22] retrospectively analyzed the CAIRO (CApecitabine, IRinotecan, and Oxaliplatin) and the CAIRO2 studies, randomized phase III clinical studies in patients with mCRC. In the CAIRO study, the authors compared the treatment group with first-line capecitabine, second-line irinotecan and third-line capecitabine plus oxaliplatin to the group with first-line capecitabine plus irinotecan and secondline capecitabine plus oxaliplatin [24]. Of the total 399 patients, there were 258 patients in the first-line surgery group and 141 patients in the first-line chemotherapy group. In the analysis of patients' characteristics, the first-line chemotherapy group showed more frequent abnormal lactate dehydrogenase (LDH), extrahepatic metastasis, and rectal and rectosigmoid colon tumors. The overall survivals and the progression free survivals (PFSs) in the first-line surgery group and the first-line chemotherapy group were 16.7 months and 11.4 months, and 6.7 month and 5.9 month, respectively, indicating that there were significant differences. In the multivariate analysis considering the patients' characteristics, resection of primary tumors was shown to be a significant prognosis factor. The frequencies of vomiting-related grade 3 to 4 toxicity were $11 \%$ and $5 \%(\mathrm{P}=0.053)$, and those of ileus-related grade 3 to 4 toxicity were $7 \%$ and $2 \%(P=0.019)$ in the first-line surgery group and the first-line chemotherapy group, respectively.

The CAIRO2 study investigated the cetuximab group in addition to the capecitabine, oxaliplatin, and bevacizumab treatment group [25]. A total of 448 patients were included: 289 and 159 patients in the first-line surgery group and the first-line chemotherapy group, respectively. In the patients' characteristics comparison, male patients were outnumbered in the first-line chemotherapy group; further, abnormal LDH, poor performance status, and metastatic lesions, including liver metastases, were more frequently shown in the first-line chemotherapy group. Overall survivals and PFSs were 20.7 months and 13.4 months, and 10.5 months and 7.8 months, respectively, which were all significantly different. In the multivariate analysis considering the patients' characteristics, resection of the primary tumor was an important prognosis factor in overall survival. Nausea $(\mathrm{P}=0.004)$, vomiting $(\mathrm{P}=0.043)$, ileus $(\mathrm{P}=0.019)$, and fatigue-related grade 3 to 4 toxicity $(\mathrm{P}=0.004)$ were all significantly higher in the first-line chemotherapy group. Thus, considering the results in both the CAIRO and the CAIRO2 studies, resection of primary tumors was the major prognosis factor in regard to improved overall survival.

The Fédération Francophone de Cancérologie Digestive (FFCD) 9601 trial, which is a randomized phase-3 clinical study, compared the efficacy of 5-FU infusion and Raltitrexed with three different schedules as a first-line chemotherapy for patients with mCRC [26]. Ferrand et al. [23] retrospectively analyzed 294 patients recruited from 39 hospitals, and 216 patients were found to be synchronous metastatic patients. One hundred fifty-six patients $(72 \%)$ had undergone a resection of the primary tumor prior to the study initiation. A significant difference was shown in the locations of the primary tumors, indicating that $14 \%$ of patients in the first-line surgery group had a tumor in the rectum as opposed to $35 \%$ in the first-line chemotherapy group. Analyzing the survival rate, the PFSs and the overall survivals of the first-line surgery group and the first-line chemotherapy group were 5.1 months and 2.9 months, and 16.3 months and 9.6 months, respectively; both survival rates were higher in the first-line surgery group than in the first-line chemotherapy group. Resection of the primary tumors was the most significant prognosis factor in the multivariate analysis, as well. The percentage of hospitalization for surgery and obstruction was $7 \%$ in both groups. Thus, in the study, resection of primary tumors was shown to improve the survival rate for synchronous mCRC patients who were under first-line, single-agent chemotherapy.

One of the major limitations of the study is that resection of primary tumors had already been determined prior to the study's initiation. Thus, clear justifications for not resecting the primary tumors could not be found. To be specific, unresectable primary tumors, poor performance status of patients, cases in which systemic chemotherapy should used due to the complications of metastatic lesions prior to the surgery, and symptoms related to the primary tumor could have been possible justifications. However, in the multivariate analysis, resection of primary tumors was found to be a significant prognosis factor in the CAIRO study, the CAIRO2 study, and the FFCD 9601 study. Furthermore, in these studies, the chemotherapy regimen did not include recent targeted agents. Regardless, the survival duration was shown to be improved, as found in Venderbosch et al. [22]'s CAIRO/CAIRO2 analysis. In summary, it was suggested that the improved survival duration was not associated with the types of chemotherapy regimens. In Stillwell et al. [27]'s meta-analysis, it was expected that resection of primary tumors would prolong by approximately 6 months the median survival duration.

Anwar et al. [28] published a systematic review of 21 studies re- 
ported between 1980 and 2010. In this systematic review, the authors compared the first-line surgery group and first-line chemotherapy group in mCRC. To evaluate the impact on the survival, the authors included asymptomatic patients. Patients undergoing non-resectional surgery such as bypass procedure or enterostomy were also included in the first line chemotherapy group. They concluded that resection of primary tumors may reduce obstruction, which requires emergency operation, and improve the survival rate. Further, delaying chemotherapy owing to the resection would not negatively affect the survival rate for patients. However, a major limitation of the study was that it was not based on a comparison of randomized clinical studies; in the multivariate prognosis-factor analysis, tumor burden and performance status were significant prognosis factors for the survival rate. The authors concluded that in stage IV colorectal cancer patients, resection of primary tumors should be determined based on tumor burden or performance status rather than symptoms.

Scheer et al. [29] made a comparison between the first-line surgery group and first-line chemotherapy group in asymptomatic stage IV colorectal cancer patients. The primary objective was to investigate tumor-related complications in the first-line chemotherapy group, and the authors suggested performing chemotherapy for asymptomatic cancer patients. However, in the study, the survival rate of the first-line surgery group was higher than that of the first-line chemotherapy group, which might be a possible limitation of the study regarding biased selection of patients.

Lastly, Stillwell et al. [27]'s meta-analysis was reviewed. In the study, the authors performed a meta-analysis of data from eight retrospective, single-institute studies that included both asymptomatic and minimally-symptomatic unresectable colorectal cancer patients. Of the 1,062 patients, the first-line surgery group and the first-line chemotherapy group included 725 and 337 patients, respectively. The median survival durations of the first-line surgery group and the first-line chemotherapy group were 14 to 23 months and 6 to 22 months, respectively; the estimated survival advantage of the first-line surgery group was 6 months. The prognosis factors related to the survival rate were tumor burden and performance status. The cases where radical resection was possible after the primary treatment for both the first-line surgery group and the first-line chemotherapy group were analyzed; $1.8 \%$ to $18.8 \%$ and $1.2 \%$ to $22 \%$ of the patients were available for the radical resection after the primary treatment in the first-line surgery group and first-line chemotherapy group, respectively. There was no difference noted between the groups. In the first-line surgery group, the complication rate after surgery was $23 \%$; the most common complication was wound infection. The mortality was $1.7 \%$. When the frequency of intestinal complications was quantitatively analyzed, the first-line chemotherapy group had a 7.3 times higher risk than the first-line surgery group.

The intestinal complication rate in the first-line chemotherapy group was $22.2 \%$, and $18.3 \%$ of the patients required the surgery with complications required surgery. Obstruction was the most common complication. Obstruction was most common in the rectum $(41.5 \%)$, followed by the left colon $(34.1 \%)$ and the right colon (24.4\%). The length of hospitalization of the first-line chemotherapy group was 11 days, which was significantly shorter than that of the first-line surgery group $(P=0.003)$. In summary, the survival rate was shown to be improved in the group with resection of the primary tumor, and no convincing evidence was found for negative impacts of chemotherapy on tumor responses or survival even though the first-line surgery group showed a longer hospitalization and had delayed chemotherapy. In addition, the authors suggested that resection of primary tumors might prevent emergency surgery for such complications as it would reduce intestinal complications.

Karoui et al. [20] pointed out that even though patients with obstruction had been treated with stent insertion, the chemotherapy would be delayed during the stent insertion and symptom development. Further, minimally-invasive surgery, such as laparoscopic surgery, has been becoming more popular for stage IV colorectal cancer patients. Because it allows faster recovery with shorter hospitalization compared to conventional laparotomy, laparoscopic surgery should be strongly considered for the patients' sake [30] (Tables 2 and 3).

\section{THE OPINION IN FAVOR OF FIRST-LINE CHEMOTHERAPY IN UNRESECTABLE SYNCHRONOUS METASTATIC COLORECTAL CANCER}

Traditionally, 5-FU was the only chemotherapeutic agent that could be administered to patients with mCRC. The response rate was approximately $20 \%$, and it was difficult to expect long-term survival for most unresectable colorectal cancer patients with liver metastases [31]. Chemotherapeutic agents, administration methods, and novel biological agents for the treatments of colorectal cancer with liver metastases allowed surgery to be performed even for initially unresectable metastatic lesions. The response rates of new cytotoxic chemotherapeutic agents such as oxaliplatin and irinotecan were improved by more than $50 \%$, and more than $38 \%$ of the patients with unresectable lesions were able to have resections $[32,33]$.

The survival rate of patients with $\mathrm{mCRC}$ has rapidly increased since 1990 because of the development of chemotherapy and surgery. The median survival duration of patients with stage IV colorectal cancer was 14.2 months in the 1990s, and it was increased to 18.6 months in early 2000s and to even 30 months recently. The five-year overall survival in stage IV patients was improved from $9.1 \%$ in the 1990 s to $19.2 \%$ in 2003 . Such an improvement in the survival rate before 2004 was due to the increase in the number of resections performed, around $20 \%$ of the patients with metastatic colorectal cancer underwent resections, whereas the results after 2004 were mainly due to new chemotherapy regimens. 
Table 2. Demographic data from selected studies

\begin{tabular}{|c|c|c|c|c|c|c|c|c|c|}
\hline \multirow{2}{*}{ Investigator } & \multirow{2}{*}{ Resection } & \multirow{2}{*}{$\begin{array}{c}\text { Chemotherapy } \\
\text { after } \\
\text { resection }\end{array}$} & \multirow{2}{*}{$\begin{array}{l}\text { Nonresection \& } \\
\text { first-line } \\
\text { chemotherapy }\end{array}$} & \multicolumn{2}{|c|}{$\begin{array}{l}\text { Right-sided colon primary } \\
\text { tumor }\end{array}$} & \multicolumn{2}{|c|}{$\begin{array}{l}>25 \% \text { Hepatic parenchymal } \\
\text { metastases }\end{array}$} & \multicolumn{2}{|c|}{$\begin{array}{l}\text { Extrahepatic } \\
\text { metastases }\end{array}$} \\
\hline & & & & Resection & Nonresection & Resection & Nonresection & Resection & Nonresection \\
\hline Ruo et al. [10] & 127 & $0(0)$ & $103^{\mathrm{a}}$ & $58(46)$ & $29(28)$ & $40(41)$ & $29(55)$ & $56(44)$ & $61(59)$ \\
\hline Galizia et al. [7] & 42 & $42(100)$ & 23 & $12(29)$ & $6(26)$ & $26(62)$ & $14(61)$ & NA & NA \\
\hline Kaufman et al. [18] & 115 & $115(100)$ & 21 & NA & NA & NA & NA & NA & NA \\
\hline Bajwa et al. [19] & 32 & $32(100)$ & 35 & $14(47)$ & $10(29)$ & NA & NA & $0(0)$ & $0(0)$ \\
\hline \multirow[t]{2}{*}{ Venderbosch et al. [22] } & 258 & $258(100)$ & 141 & $183(71)^{\mathrm{b}}$ & $78(55)^{b}$ & NA & NA & $23(9)$ & $26(19)$ \\
\hline & 289 & $289(100)$ & 159 & $128(51)$ & $71(48)$ & NA & NA & $34(12)$ & $4(3)$ \\
\hline Karoui et al. [20] & 85 & $84(99)$ & 123 & $30(35)$ & $37(30)$ & NA & NA & $34(40)$ & $47(38)$ \\
\hline Ferrand et al. [23] & 216 & $156(72)$ & 60 & $52(34)$ & $10(17)$ & NA & NA & NA & NA \\
\hline Scoggins et al. [44] & 66 & $0(0)$ & $23^{\mathrm{a}}$ & $31(47)$ & $6(26)$ & $18(32)$ & $8(40)$ & $10(15)$ & $9(39)$ \\
\hline Tebbutt et al. [43] & 280 & $280(100)$ & 82 & $188(67)^{b}$ & $44(54)^{b}$ & NA & NA & $56(20)^{c}$ & $11(13)^{c}$ \\
\hline Michel et al. [9] & 31 & 30 (97) & 23 & $28(90)^{b}$ & $15(65)^{b}$ & $11(36)^{d}$ & $9(39)^{d}$ & NA & NA \\
\hline Benoist et al. [12] & 32 & 30 (94) & 27 & $9(28)$ & $9(33)$ & $27(84)$ & 24 (89) & $2(6)$ & $2(7)$ \\
\hline Seo et al. [52] & 144 & $144(100)$ & 83 & $30(21)$ & $26(31)$ & $75(52)^{d}$ & $50(60)^{d}$ & NA & NA \\
\hline McCahill et al. [53] & NA & NA & 86 & NA & NA & NA & NA & NA & NA \\
\hline
\end{tabular}

Values are presented as number (\%).

NA, not available.

ancluding chemoradiation. ${ }^{\mathrm{b}} \mathrm{C}$ olon. ${ }^{\mathrm{c} P e r i t o n e a l / o m e n t a l ~ m e t a s t a s i s . ~}{ }^{\mathrm{d}}$ Five liver metastases or more.

A retrospective study was carried out based on SEER data and based on data on patients who received treatment for metastatic colorectal cancer at the MD Anderson Cancer Center and the Mayo Clinic in the US from 1990 to 2005 [34]. Of the 2,470 patients with $\mathrm{mCRC}$, no significant difference was observed in the results between the Mayo Clinic $(\mathrm{n}=856)$ and the MD Anderson Cancer Center $(\mathrm{n}=1,614)(\mathrm{P}=0.58)$. Overall median survival durations were similar from 1990 to 1997, but the second half of the study period, the five-year overall survival duration was significantly increased (14.2 months). Such improvements of the survival rate in those two institutes showed a pattern similar to that seen in the study done by SEER with 49,459 metastatic colorectal cancer patients from 1990 to 2005. The median survival duration was increased by 8-9 months until 2003 and then by 11 months in 2004-2005. The five-year overall survival duration was also dramatically increased. This indicates that most of the patients with mCRC lived for a long period of time even though complete recovery was not possible. Practically, since 2005, more than $30 \%$ of the patients who were diagnosed with mCRC have survived for more than 5 years.

Moreover, a couple of studies found that the response rate, the survival rate, and the disease-free survival rate were improved in patients with stage IV colorectal cancer by utilizing bevacizumab, the monoclonal antibody against VEGF, a targeted therapy drug [35-37]. The median survival duration increased to 2 years because of the development of chemotherapy agents $[38,39]$. In addition to the median survival duration, chemotherapy greatly re- duced not only hepatic metastasis size but also the size of primary tumors. Both metastatic lesions and primary tumors showed responses with chemotherapy only. Furthermore, primary tumors have shown complete responses to new cytotoxic and targeted agents [40].

Poultsides et al. [41] investigated the complications caused by primary tumors when first-line chemotherapy was performed in 233 stage IV colorectal cancer patients. Oxaliplatin- or irinotecanbased triple-drug chemotherapy (fluorouracil, leucovorin) with or without bevacizumab was utilized as the chemotherapy regimen. Emergency surgery for obstruction and perforation due to primary tumors was required in 16 patients (7\%). A total of 213 patients $(89 \%)$ did not require any surgical and nonsurgical treatments for primary tumors. Another animal study reported that surgical resection altered the immune responses in patients, thereby facilitating the growth of tumors after surgery [42]. Moreover, a couple of recent studies reported that a resection of the primary tumor was not required in cases of intact primary tumors for stage IV colorectal cancer. According to those studies, surgical resection was necessary in less than $15 \%$ of the cases while the remaining $85 \%$ of the cases did not require a resection [41, 43-46]. Additionally, even though palliative resection of the primary tumor was carried out for $\mathrm{mCRC}$, complications related to the large intestine could occur later, and surgery should be applied in some cases $[43,47]$. Based on these studies, the resection rate of primary tumors has decreased gradually [5].

The opinion in favor of first-line chemotherapy indicated that 
Table 3. Outcome data from selected studies

\begin{tabular}{|c|c|c|c|c|c|c|c|c|}
\hline \multirow{2}{*}{ Investigator } & \multirow{2}{*}{$\begin{array}{l}\text { Complications } \\
\text { after resection }\end{array}$} & \multirow{2}{*}{$\begin{array}{l}\text { Mortality after } \\
\text { resection }\end{array}$} & \multirow{2}{*}{$\begin{array}{l}\text { Complications after } \\
\text { nonresection }\end{array}$} & \multicolumn{3}{|c|}{ Survival (mo) } & \multirow{2}{*}{$\begin{array}{l}\text { Chemotherapy } \\
\text { regimen }\end{array}$} & \multirow{2}{*}{$\begin{array}{l}\text { Follow-up } \\
\text { (mo) }\end{array}$} \\
\hline & & & & Resection & Nonresection & Significance & & \\
\hline Ruo et al. [10] & $26(20.5)$ & $2(1.6)$ & $30(29)$ & 16 & 9 & $<0.001$ & NA & NA \\
\hline Kaufman et al. [18] & NA & NA & NA & 22 & 15 & NA & NA & NA \\
\hline \multirow[t]{2}{*}{ Venderbosch et al. [22] } & NA & NA & NA & 16.7 & 11.4 & $<0.001$ & $\begin{array}{l}\text { Capecitabine, oxaliplatin, } \\
\text { irinotecan }\end{array}$ & 31.5 \\
\hline & NA & NA & NA & 20.7 & 13.4 & $<0.001$ & $\begin{array}{l}\text { Capecitabine, oxaliplatin, } \\
\text { bevacizumab, cetuximab }\end{array}$ & 23 \\
\hline Karoui et al. [20] & NA & NA & $23(19)$ & 30.7 & 21.9 & 0.031 & $\begin{array}{l}\text { Oxaliplatin, irinotecan, } \\
\text { bevacizumab, cetuximab }\end{array}$ & 19.7 \\
\hline Tebbutt et al. [43] & $37(13.2)^{b}$ & $N A$ & $11(13.4)^{b}$ & 14 & 8.2 & NS & 5-FU-based, other & 30 (R), 19 (NR) \\
\hline Michel et al. [9] & NA & $0(0)$ & $5(21.7)$ & 21 & 14 & NS & Oxaliplatin, irinotecan & NA \\
\hline Benoist et al. [12] & $6(19)$ & $0(0)$ & $4(14.8)$ & 23 & 22 & NS & $\begin{array}{l}\text { 5-FU-leucovorin, oxaliplatin, } \\
\text { irinotecan, other }\end{array}$ & 24 \\
\hline Seo et al. [52] & $29(20.2)^{c}$ & $0(0)$ & $17(20.5)^{c}$ & 22 & 14 & NS & $\begin{array}{l}\text { Fluoropyrimidine, oxaliplatin, } \\
\text { irinotecan, bevacizumab, } \\
\text { or cetuximab }\end{array}$ & 49 \\
\hline McCahill et al. [53] & NA & NA & $12(14)$ & NA & 19.9 & NA & $\begin{array}{l}\text { Oxaliplatin, Leucovorin, 5-FU, } \\
\text { bevacizumab }\end{array}$ & 20.4 \\
\hline
\end{tabular}

Values are presented as number (\%).

NA, not available; 5-FU, 5-fluorouracil; R, resection; NR, nonresection; NS, not significant.

${ }^{a}$ Admission for surgery or bowel obstruction. 'Intestinal obstruction. Intestinal complication.

the use of systemic chemotherapy would be delayed when a resection had been performed and that, sometimes, chemotherapy would be unavailable due to the complications associated with surgery. Early use, as compared to later use, of palliative chemotherapy is advantageous for the quality of life and the overall survival [48]. Because the occurrences of the symptoms caused by primary tumors are not very high in most cases, it has been pointed out that surgical resection is unnecessary. In some cases, a hepatectomy is performed at first in colorectal cancer patients with hepatic metastasis because the incidence of complications is low in cases of asymptomatic colorectal cancer [49-51]. To date, however, such data have not been very reliable because there have been no prospective comparison studies with appropriate designs.

Scoggins et al. [44] analyzed 89 stage IV colorectal cancer patients. The first-line surgery group for primary tumors and the first-line chemotherapy group contained 66 and 23 patients, respectively. In the first-line surgery group, the incidence of complications after surgery was $30.3 \%$, and the mortality rate was $4.6 \%$. Wound infection and sepsis were the most common complications. Only 2 cases $(8.7 \%)$ in the first-line chemotherapy group showed obstruction caused by the primary tumors. No significant difference was observed in survival duration between the firstline surgery group (14.5 months) and the first-line chemotherapy group (16.6 months) $(\mathrm{P}=0.59)$.

Tebutt et al. [43] investigated the incidence of intestinal complications in 362 patients with unresectable colorectal cancer. Two hundred eighty patients and 82 patients were in the first-line surgery group and the first-line chemotherapy group, respectively. Obstruction was exhibited in 37 patients in the first-line surgery group (13.2\%) and in 11 patients in the first-line chemotherapy group (13.4\%), but the difference was not significant. However, 14 patients in the first-line surgery group (38\%) required surgical treatments because of obstruction whereas 8 patients in the firstline chemotherapy group (73\%) requested surgical treatments. Peritonitis, fistula formation, and intestinal hemorrhage were observed in $2.4 \%, 3.7 \%$, and $3.7 \%$ of the patients in the first-line chemotherapy group, respectively. The median survival durations were 14 months and 8.2 months for the first-line surgery group and the first-line chemotherapy group, respectively, but the difference was not statistically significant. 
Michel et al. [9] analyzed a total of 44 patients with mCRC. There were 31 patients in the first-line surgery group and 23 patients in the first-line chemotherapy group. Rectal cancer was rarely observed in the first-line surgery group, but more than at least three hepatic metastases was exhibited in many cases. The median survival durations were 21 months and 14 months for the first-line surgery group and the first-line chemotherapy group, respectively, but this difference was not significant $(\mathrm{P}=0.718)$. In the first-line chemotherapy group, $21.7 \%$ of the patients required surgical treatments due to obstruction.

Benoist et al. [12] performed a case-matched study of 59 colorectal cancer patients with unresectable hepatic metastasis. There were 27 patients in the first-line surgery group and 32 patients in the first-line chemotherapy group. The median survival durations were 22 months and 23 months for the first-line surgery group and the first-line chemotherapy group, respectively, but no statistical difference was indicated. Of the first-line surgery group, $19 \%$ showed complications after the surgery while $14.8 \%$ of the first-line chemotherapy group showed complications associated with the primary tumors. A significant difference was observed in the lengths of hospitalization, 22 days for the first-line surgery group and 11 days for the first-line chemotherapy group.

Seo et al. [52] investigated 227 patients with unresectable stage IV colorectal cancer. A total of 144 and 83 patients were included in the first-line surgery group and the first-line chemotherapy group, respectively. Major intestinal complications, including obstruction, peritonitis, fistula formation, and bleeding, were exhibited in $20.2 \%$ of the first-line surgery group and $20.5 \%$ of the firstline chemotherapy group, but this difference was not significant. However, a significant difference was found in the initiation time of chemotherapy, which was 37 days for the first-line surgery group and 7 days for the first-line chemotherapy group $(\mathrm{P}<$ 0.001). The median survival durations of the first-line surgery group and the first-line chemotherapy group were 22 months and 14 months, respectively, but no statistically significant difference was indicated. In the multivariate analysis, high grade differentiation and more than five hepatic metastatic lesions were unfavorable prognosis factors while the utilization of oxaliplatin was related to a favorable survival rate.

Recently, the results of the National Surgical Adjuvant Breast and Bowel Project (NSABP) Trial C-10 were published [53]. This study was a phase-2 clinical trial to investigate the safety of nonsurgical treatment when unresectable stage IV colorectal cancer patients with asymptomatic primary tumors were treated using 5-Fluorouracil, Leucovorin, and Oxaliplatin (mFOLFOX6) chemotherapy drugs with Bevacizumab. The patients in ECOG performance status $0-1$, excluding rectal cancer, participated in the project. The project was not focused to compare the first-line surgery group and the first-line chemotherapy group regarding primary tumors. The main objective of the study was to analyze patients for whom surgery was needed due to major morbidity events, such as bleeding, perforation, obstruction, and fistula for- mation, caused by primary tumors. The study was carried out with 86 patients in 29 hospitals. The median follow-up duration was 20.7 months, and major morbidity events were shown in 12 patients (14\%). Of those 12 patients, 10 underwent surgery owing to obstruction (8 cases), perforation ( 1 case), and abdominal pain ( 1 case); and the other 2 patients expired. Of the 10 patients who underwent surgery, 6 involved urgency while 4 did not; 1 patient (10\%) died after the surgery. In chemotherapy-related cases, with a 19.9-month median survival duration, 4 patients expired. The cumulative incidence of major morbidity at the 24th month was $16.4 \%$; chemotherapy with mFOLFOX6 and bevacizumab was carried out in the remaining $83.6 \%$ without major complications due to the primary tumors. Therefore, the study suggested that the resection of asymptomatic primary tumors was not necessary prior to chemotherapy. Regarding the clinical problems when administering bevacizumab, previous studies reported that $3.1 \%$ to $5.1 \%$ moderate or severe bleeding occurred. Although perforation was not frequently encountered (approximately 1.5\%), careful attention was still required $[5,35,36]$. In the NSABP study, on the other hand, because only two patients showed perforation, administration of bevacizumab was considered to be safe.

Regarding the result of NSABP Trial C-10, Chang [54] pointed out that asymptomatic primary tumors were not clearly defined. It was reported that the late onset caused by the progression of the tumors or intestinal stenosis after first-line chemotherapy had not been well known. Temple et al. [6] pointed out that the mortality after primary tumor resection, $9 \%$, was elevated by $26 \%$ when surgery was performed after chemotherapy. In a study by Poultsides et al. in 2009 [41], the mortality after emergency resection was also very high (12.5\%). Therefore, the authors suggested that colonoscopy on a regular basis to diagnose in advance the complications associated with the primary tumors would be useful in order to avoid emergency situation.

In 2008, a systematic review was carried out by Scheer et al. [29] based upon the data on 850 patients from 7 papers published from 1999 to 2006 . When first-line chemotherapy was performed in unresectable stage IV colorectal cancer patients, obstruction caused by primary tumors and intestinal hemorrhage were observed in $13.9 \%$ and $3 \%$ of the patients, respectively. On the other hand, when first-line surgery for primary tumors was carried out, complications were frequent, $18.8 \%$ to $47 \%$, after the surgery. Thus, the researchers recommended implementing chemotherapy first. Furthermore, they mentioned that the patients who possessed asymptomatic primary tumors died due to metastasis and the progression of the carcinoma; on the other hand, it would be very rare to die because of the complications related to primary tumors [55].

Damjanov et al. [3] suggested that chemotherapy including new targeted therapy drugs increased response rates of metastatic lesions, as well as primary tumors [56]. Moreover, intestinal complications, such as obstruction due to primary tumors and intestinal hemorrhage, could be treated by using endoscopic stent inser- 
tion and hemostatic treatment. Therefore, the authors strongly recommended performing chemotherapy first, instead of resection, in patients having unresectable stage IV colorectal cancer with asymptomatic primary tumors.

In 2012, the Cochrane collaboration analyzed 7 studies with a total of 1,086 patients, including 722 in the first-line surgery group and 364 in the first-line chemotherapy group [57]. It was reported that there was no difference in overall survival between those two groups was reported. Also, no sufficient reasons were found to support the conclusion that surgical resection reduced the complications associated with primary tumors. However, as the review paper was a retrospective, single-institute study, prospective comparison studies are warranted in the future. Additionally, three ongoing studies were introduced:

1) Chemotherapy with or without surgery in treating patients with mCRC that cannot be removed by surgery (ClinicalTrials. gov study no. NCT01086618):

Purpose: To determine whether overall survival is improved in patients with asymptomatic, unresectable metastatic colorectal cancer treated with chemotherapy alone versus surgery followed by chemotherapy.

2) The SUPER Study: A randomized phase III multicenter trial evaluating the role of palliative surgical resection of the primary tumors in patients with mCRC (ACTRN12609000680268):

Purpose: To determine whether surgical resection of the primary tumors in patients with stage IV colorectal cancer decreases intestinal complications and improves overall survival and quality of life.

3) Resection of the primary tumors versus no resection prior to systemic therapy in patients with colon cancer and synchronous unresectable metastases (Union for International Cancer Control stage IV): a randomized controlled multicenter trial (ISRCTN30964555):

Purpose: To evaluate the efficacy of primary tumor resection prior to systemic chemotherapy to prolong survival in patients with newly diagnosed colon cancer who are not amenable to curative therapy [58].

\section{CONCLUSION}

Disagreements exist as to whether the appropriate primary treatment for unresectable stage IV colorectal cancer patients with asymptomatic primary tumors is surgical resection or chemotherapy. Prospective comparison studies only focusing on first-line treatment modalities have not been performed yet; thus, future clinical studies with objectives regarding survival duration and quality of life should be implemented as soon as possible. In addition to clinical studies, multidisciplinary team approaches would be helpful in choosing the appropriate therapy. Regardless of symptoms, the performance status and the tumor burden should be considered as well. When performing surgical resection, minimally invasive approaches, such as laparoscopic surgery, should be considered for the patients' sake.

\section{CONFLICT OF INTEREST}

No potential conflict of interest relevant to this article was reported.

\section{REFERENCES}

1. Kim DH. Risk factors of colorectal cancer. J Korean Soc Coloproctol 2009;25:356-62.

2. Jung KW, Park S, Kong HJ, Won YJ, Lee JY, Seo HG, et al. Cancer statistics in Korea: incidence, mortality, survival, and prevalence in 2009. Cancer Res Treat 2012;44:11-24.

3. Damjanov N, Weiss J, Haller DG. Resection of the primary colorectal cancer is not necessary in nonobstructed patients with metastatic disease. Oncologist 2009;14:963-9.

4. National Comprehensive Cancer Network. NCCN clinical practice guidelines in oncology (NCCN guidelines): colon/rectal cancer. ver. 1. 2010 [Internet]. Fort Wathington: NCCN; c2012 [cited 2013 Jan 14]. Available from: http://www.nccn.org/professionals/ physician_gls/f_guidelines.asp.

5. Cook AD, Single R, McCahill LE. Surgical resection of primary tumors in patients who present with stage IV colorectal cancer: an analysis of surveillance, epidemiology, and end results data, 1988 to 2000. Ann Surg Oncol 2005;12:637-45.

6. Temple LK, Hsieh L, Wong WD, Saltz L, Schrag D. Use of surgery among elderly patients with stage IV colorectal cancer. J Clin Oncol 2004;22:3475-84.

7. Galizia G, Lieto E, Orditura M, Castellano P, Imperatore V, Pinto $\mathrm{M}$, et al. First-line chemotherapy vs bowel tumor resection plus chemotherapy for patients with unresectable synchronous colorectal hepatic metastases. Arch Surg 2008;143:352-8.

8. Law WL, Chan WF, Lee YM, Chu KW. Non-curative surgery for colorectal cancer: critical appraisal of outcomes. Int J Colorectal Dis 2004;19:197-202.

9. Michel P, Roque I, Di Fiore F, Langlois S, Scotte M, Teniere P, et al. Colorectal cancer with non-resectable synchronous metastases: should the primary tumor be resected? Gastroenterol Clin Biol 2004;28:434-7.

10. Ruo L, Gougoutas C, Paty PB, Guillem JG, Cohen AM, Wong WD. Elective bowel resection for incurable stage IV colorectal cancer: prognostic variables for asymptomatic patients. J Am Coll Surg 2003;196:722-8.

11. Law WL, Chu KW. Outcomes of resection of stage IV rectal cancer with mesorectal excision. J Surg Oncol 2006;93:523-8.

12. Benoist S, Pautrat K, Mitry E, Rougier P, Penna C, Nordlinger B. Treatment strategy for patients with colorectal cancer and synchronous irresectable liver metastases. Br J Surg 2005;92:1155-60.

13. Joffe J, Gordon PH. Palliative resection for colorectal carcinoma. Dis Colon Rectum 1981;24:355-60.

14. Stearns MW Jr, Binkley GE. Palliative surgery for cancer of the 
rectum and colon. Cancer 1954;7:1016-9.

15. Modlin J, Walker HS. Palliative resections in cancer of the colon and rectum. Cancer 1949;2:767-76.

16. Costi R, Mazzeo A, Di Mauro D, Veronesi L, Sansebastiano G, Violi V, et al. Palliative resection of colorectal cancer: does it prolong survival? Ann Surg Oncol 2007;14:2567-76.

17. Sorbye H, Kohne CH, Sargent DJ, Glimelius B. Patient characteristics and stratification in medical treatment studies for metastatic colorectal cancer: a proposal for standardization of patient characteristic reporting and stratification. Ann Oncol 2007;18:1666-72.

18. Kaufman MS, Radhakrishnan N, Roy R, Gecelter G, Tsang J, Thomas $\mathrm{A}$, et al. Influence of palliative surgical resection on overall survival in patients with advanced colorectal cancer: a retrospective single institutional study. Colorectal Dis 2008;10:498-502.

19. Bajwa A, Blunt N, Vyas S, Suliman I, Bridgewater J, Hochhauser D, et al. Primary tumour resection and survival in the palliative management of metastatic colorectal cancer. Eur J Surg Oncol 2009;35:164-7.

20. Karoui M, Roudot-Thoraval F, Mesli F, Mitry E, Aparicio T, Des Guetz G, et al. Primary colectomy in patients with stage IV colon cancer and unresectable distant metastases improves overall survival: results of a multicentric study. Dis Colon Rectum 2011;54: 930-8.

21. Hapani S, Chu D, Wu S. Risk of gastrointestinal perforation in patients with cancer treated with bevacizumab: a meta-analysis. Lancet Oncol 2009;10:559-68.

22. Venderbosch S, de Wilt JH, Teerenstra S, Loosveld OJ, van Bochove A, Sinnige HA, et al. Prognostic value of resection of primary tumor in patients with stage IV colorectal cancer: retrospective analysis of two randomized studies and a review of the literature. Ann Surg Oncol 2011;18:3252-60.

23. Ferrand F, Malka D, Bourredjem A, Allonier C, Bouche O, Louafi $S$, et al. Impact of primary tumour resection on survival of patients with colorectal cancer and synchronous metastases treated by chemotherapy: results from the multicenter, randomised trial Fédération Francophone de Cancérologie Digestive 9601. Eur J Cancer 2013;49:90-7.

24. Koopman M, Antonini NF, Douma J, Wals J, Honkoop AH, Erdkamp FL, et al. Sequential versus combination chemotherapy with capecitabine, irinotecan, and oxaliplatin in advanced colorectal cancer (CAIRO): a phase III randomised controlled trial. Lancet 2007;370:135-42.

25. Tol J, Koopman M, Rodenburg CJ, Cats A, Creemers GJ, Schrama JG, et al. A randomised phase III study on capecitabine, oxaliplatin and bevacizumab with or without cetuximab in first-line advanced colorectal cancer, the CAIRO2 study of the Dutch Colorectal Cancer Group (DCCG). An interim analysis of toxicity. Ann Oncol 2008;19:734-8.

26. Ducreux M, Bouche O, Pignon JP, Mousseau M, Raoul JL, Cassan $\mathrm{P}$, et al. Randomised trial comparing three different schedules of infusional 5FU and raltitrexed alone as first-line therapy in metastatic colorectal cancer. Final results of the Fédération Franco- phone de Cancérologie Digestive (FFCD) 9601 trial. Oncology 2006;70:222-30.

27. Stillwell AP, Buettner PG, Ho YH. Meta-analysis of survival of patients with stage IV colorectal cancer managed with surgical resection versus chemotherapy alone. World J Surg 2010;34:797807.

28. Anwar S, Peter MB, Dent J, Scott NA. Palliative excisional surgery for primary colorectal cancer in patients with incurable metastatic disease. Is there a survival benefit? A systematic review. Colorectal Dis 2012;14:920-30.

29. Scheer MG, Sloots CE, van der Wilt GJ, Ruers TJ. Management of patients with asymptomatic colorectal cancer and synchronous irresectable metastases. Ann Oncol 2008;19:1829-35.

30. Allaix ME, Degiuli M, Giraudo G, Marano A, Morino M. Laparoscopic versus open colorectal resections in patients with symptomatic stage IV colorectal cancer. Surg Endosc 2012;26:2609-16.

31. Giacchetti S, Itzhaki M, Gruia G, Adam R, Zidani R, Kunstlinger F, et al. Long-term survival of patients with unresectable colorectal cancer liver metastases following infusional chemotherapy with 5-fluorouracil, leucovorin, oxaliplatin and surgery. Ann Oncol 1999;10:663-9.

32. Adam R, Delvart V, Pascal G, Valeanu A, Castaing D, Azoulay D, et al. Rescue surgery for unresectable colorectal liver metastases downstaged by chemotherapy: a model to predict long-term survival. Ann Surg 2004;240:644-57.

33. Alberts SR, Horvath WL, Sternfeld WC, Goldberg RM, Mahoney MR, Dakhil SR, et al. Oxaliplatin, fluorouracil, and leucovorin for patients with unresectable liver-only metastases from colorectal cancer: a North Central Cancer Treatment Group phase II study. J Clin Oncol 2005;23:9243-9.

34. Kopetz S, Chang GJ, Overman MJ, Eng C, Sargent DJ, Larson DW, et al. Improved survival in metastatic colorectal cancer is associated with adoption of hepatic resection and improved chemotherapy. J Clin Oncol 2009;27:3677-83.

35. Hurwitz H, Fehrenbacher L, Novotny W, Cartwright T, Hainsworth J, Heim W, et al. Bevacizumab plus irinotecan, fluorouracil, and leucovorin for metastatic colorectal cancer. N Engl J Med 2004;350: 2335-42.

36. Kabbinavar FF, Schulz J, McCleod M, Patel T, Hamm JT, Hecht $\mathrm{JR}$, et al. Addition of bevacizumab to bolus fluorouracil and leucovorin in first-line metastatic colorectal cancer: results of a randomized phase II trial. J Clin Oncol 2005;23:3697-705.

37. Grothey A, Sugrue MM, Purdie DM, Dong W, Sargent D, Hedrick $\mathrm{E}$, et al. Bevacizumab beyond first progression is associated with prolonged overall survival in metastatic colorectal cancer: results from a large observational cohort study (BRiTE). J Clin Oncol 2008;26:5326-34.

38. de Gramont A, Figer A, Seymour M, Homerin M, Hmissi A, Cassidy J, et al. Leucovorin and fluorouracil with or without oxaliplatin as first-line treatment in advanced colorectal cancer. J Clin Oncol 2000;18:2938-47.

39. Douillard JY, Cunningham D, Roth AD, Navarro M, James RD, 
Karasek P, et al. Irinotecan combined with fluorouracil compared with fluorouracil alone as first-line treatment for metastatic colorectal cancer: a multicentre randomised trial. Lancet 2000;355: 1041-7.

40. Karoui M, Koubaa W, Delbaldo C, Charachon A, Laurent A, Piedbois $\mathrm{P}$, et al. Chemotherapy has also an effect on primary tumor in colon carcinoma. Ann Surg Oncol 2008;15:3440-6.

41. Poultsides GA, Servais EL, Saltz LB, Patil S, Kemeny NE, Guillem JG, et al. Outcome of primary tumor in patients with synchronous stage IV colorectal cancer receiving combination chemotherapy without surgery as initial treatment. J Clin Oncol 2009; 27:3379-84.

42. Carter JJ, Feingold DL, Kirman I, Oh A, Wildbrett P, Asi Z, et al. Laparoscopic-assisted cecectomy is associated with decreased formation of postoperative pulmonary metastases compared with open cecectomy in a murine model. Surgery 2003;134:432-6.

43. Tebbutt NC, Norman AR, Cunningham D, Hill ME, Tait D, Oates $\mathrm{J}$, et al. Intestinal complications after chemotherapy for patients with unresected primary colorectal cancer and synchronous metastases. Gut 2003;52:568-73.

44. Scoggins CR, Meszoely IM, Blanke CD, Beauchamp RD, Leach $\mathrm{SD}$. Nonoperative management of primary colorectal cancer in patients with stage IV disease. Ann Surg Oncol 1999;6:651-7.

45. Mahteme H, Pahlman L, Glimelius B, Graf W. Prognosis after surgery in patients with incurable rectal cancer: a population-based study. Br J Surg 1996;83:1116-20.

46. Crane CH, Janjan NA, Abbruzzese JL, Curley S, Vauthey J, Sawaf $\mathrm{HB}$, et al. Effective pelvic symptom control using initial chemoradiation without colostomy in metastatic rectal cancer. Int J Radiat Oncol Biol Phys 2001;49:107-16.

47. Makela J, Haukipuro K, Laitinen S, Kairaluoma MI. Palliative operations for colorectal cancer. Dis Colon Rectum 1990;33:846-50.

48. Simmonds PC. Palliative chemotherapy for advanced colorectal cancer: systematic review and meta-analysis. Colorectal Cancer Collaborative Group. BMJ 2000;321:531-5.

49. van der Pool AE, de Wilt JH, Lalmahomed ZS, Eggermont AM, Ijzermans JN, Verhoef C. Optimizing the outcome of surgery in patients with rectal cancer and synchronous liver metastases. $\mathrm{Br} \mathrm{J}$ Surg 2010;97:383-90.
50. Mentha G, Roth AD, Terraz S, Giostra E, Gervaz P, Andres A, et al. 'Liver first' approach in the treatment of colorectal cancer with synchronous liver metastases. Dig Surg 2008;25:430-5.

51. Verhoef C, van der Pool AE, Nuyttens JJ, Planting AS, Eggermont AM, de Wilt JH. The "liver-first approach" for patients with locally advanced rectal cancer and synchronous liver metastases. Dis Colon Rectum 2009;52:23-30.

52. Seo GJ, Park JW, Yoo SB, Kim SY, Choi HS, Chang HJ, et al. Intestinal complications after palliative treatment for asymptomatic patients with unresectable stage IV colorectal cancer. J Surg Oncol 2010;102:94-9.

53. McCahill LE, Yothers G, Sharif S, Petrelli NJ, Lai LL, Bechar N, et al. Primary mFOLFOX6 plus bevacizumab without resection of the primary tumor for patients presenting with surgically unresectable metastatic colon cancer and an intact asymptomatic colon cancer: definitive analysis of NSABP trial C-10. J Clin Oncol 2012;30:3223-8.

54. Chang GJ. Challenge of primary tumor management in patients with stage IV colorectal cancer. J Clin Oncol 2012;30:3165-6.

55. Sarela A, O'Riordain DS. Rectal adenocarcinoma with liver metastases: management of the primary tumour. Br J Surg 2001;88: $163-4$.

56. Chau I, Brown G, Cunningham D, Tait D, Wotherspoon A, Norman AR, et al. Neoadjuvant capecitabine and oxaliplatin followed by synchronous chemoradiation and total mesorectal excision in magnetic resonance imaging-defined poor-risk rectal cancer. J Clin Oncol 2006;24:668-74.

57. Cirocchi R, Trastulli S, Abraha I, Vettoretto N, Boselli C, Montedori A, et al. Non-resection versus resection for an asymptomatic primary tumour in patients with unresectable stage IV colorectal cancer. Cochrane Database Syst Rev 2012;8:CD008997.

58. Rahbari NN, Lordick F, Fink C, Bork U, Stange A, Jager D, et al. Resection of the primary tumour versus no resection prior to systemic therapy in patients with colon cancer and synchronous unresectable metastases (UICC stage IV): SYNCHRONOUS: a randomised controlled multicentre trial (ISRCTN30964555). BMC Cancer 2012;12:142. 